\title{
Regrowth of Quantum Cascade Laser Active Regions on Metamorphic Buffer Layers
}

\author{
A. Rajeev ${ }^{\text {a,* }}$, L.J. Mawst ${ }^{\mathrm{a}}$, J.D. Kirch ${ }^{\mathrm{a}}$, D. Botez ${ }^{\mathrm{a}}$, J. Miao ${ }^{\mathrm{b}}$, P. Buelow ${ }^{\mathrm{b}}$, T.F. Kuech ${ }^{\mathrm{b}}$, \\ Xiaoqing Li ${ }^{c}$, C. Sigler ${ }^{a}$, S.E. Babcock ${ }^{c}$, T. Earles ${ }^{d}$
}

\begin{abstract}
:
Metamorphic buffer layers (MBLs) were used as substrates with lattice constants selected for designing and fabricating intersubband transition sources involving strained superlattices (SLs) such as Quantum Cascade Lasers (QCLs). Chemical mechanical planarization (CMP) was used to prepare the InGaAs-based MBLs for epitaxial growth. Indium enrichment of the InGaAs layer on the MBL surfaces was observed when annealed at the regrowth temperatures. This postanneal enhancement was eliminated by including a wet-etch treatment after CMP, which results in an epi-ready surface for regrowth. Ten stages of a QCL core region structure, designed for emission at a $3.4 \mu \mathrm{m}$ wavelength are regrown on a surface-optimized MBL. Such structures exhibit well defined X-ray diffraction pendellösung fringes, and transmission electron microscopy confirms planar superlattice interfaces with layer thicknesses that are in good agreement with the design target.
\end{abstract}

Keywords: A3. Superlattice, B3. Quantum cascade lasers, A3. Metalorganic vapor phase epitaxy, B2. Semiconducting III-V materials 


\section{Introduction}

Metamorphic buffer layers (MBLs) can serve as a "virtual substrate" with a designer-chosen surface lattice constant, thus expanding the compositional-design space for a variety of device structures, including short-wavelength $(<3.5 \mu \mathrm{m})$ Quantum Cascade Lasers (QCLs) [1, 2]. However, the strain relaxation, which occurs within the MBL, gives rise to a rough cross-hatched surface morphology [3] which may impact subsequently grown device structures. Chemical mechanical planarization (CMP) followed by wet-chemical etching of the MBL, is shown here to allow for the regrowth of highly-planar strained-layer superlattice (SL) structures applicable to QCLs.

\section{Experimental Procedure}

The MBLs employed for the QCL-device structures were grown by hydride vapor phase epitaxy (HVPE). The $\operatorname{In}_{\mathrm{x}} \mathrm{Ga}_{1-\mathrm{x}} \mathrm{As}$ MBLs were grown on (001) GaAs substrates with nine individual layers, each being $\sim 1.0 \mu \mathrm{m}$ thick and consisting of a fixed In composition which was incremented in steps from layer to layer. Finally, a tenth layer of final uniform composition (here: $\operatorname{~}_{0.23} \mathrm{Ga}_{0.77} \mathrm{As}$ ) and $\sim 15 \mu \mathrm{m}$ thickness is grown on the top, commonly referred to as the cap layer [4]. The buffer structure allows the formation of misfit dislocations as a means to relieve film stress while minimizing threading dislocations. The MBLs were characterized using highresolution X-ray diffraction (HR-XRD) and reciprocal space mapping (RSM). The MBL cap is found to be nearly fully relaxed (> 95\%) owing to the thickness and can exhibit a crystallographic tilt with respect to the substrate, which is a function of composition and thickness $[2,3]$. 
The MBL's InGaAs top layer of constant Indium composition displays some pits, hillocks and crosshatching. CMP employing a solution of $4 \%$ bleach and $96 \%$ silica nanoparticle slurry (50 weight $\%$ silica) was used to planarize the MBL surface by removing approximately 4-5 $\mu \mathrm{m}$ of material from the cap layer. The MBLs were cut into $1 \mathrm{~cm}^{2}$ pieces and mounted on a 2" Si wafer. The applied pressure on the samples, besides the polishing solution, was the main determinant of the polishing rate and surface morphology. The CMP process optimization for ternary materials such as the InGaAs MBL cap layer is discussed in detail in [5].

After polishing, $\operatorname{In}_{\mathrm{x}} \mathrm{Ga}_{1-\mathrm{x}} \mathrm{As} / \mathrm{Al}_{\mathrm{y}} \mathrm{In}_{1-\mathrm{y}} \mathrm{As}$ strained-layer superlattice (SL) structures were grown by metalorganic vapor phase epitaxy (MOVPE) on the prepared HVPE-grown MBL. Details of the MOVPE growth have been published previously [2]. The resulting SL structures were characterized by HR-XRD RSM, TEM imaging and atomic force microscopy (AFM) surface imaging.

\section{Results and Discussion}

\subsection{MBL Surface Preparation}

AFM measurements indicate that the RMS surface roughness of the MBL can be reduced by more than a factor of 2.5 using CMP on the as-grown MBLs which lessens the cross-hatch features and hillocks [6]. The samples subjected to CMP possessed a mound-like surface morphology, shown in Fig 1(b), after being heated within the MOVPE reactor under $\mathrm{AsH}_{3} / \mathrm{H}_{2}$ at temperatures ranging from 500 to $650{ }^{\circ} \mathrm{C}$. Upon characterization with scanning electron microscopy (SEM) using a backscatter electron detector and X-ray photoelectron spectroscopy (XPS), these mound-like features were found to possess higher mass density than the surrounding material as well as an increased $\mathrm{In} / \mathrm{Ga}$ ratio. Analysis using transmission electron 
microscopy (TEM) in the $\{220\}$ dark field condition confirmed the mound-like features to be Inrich $\operatorname{In}_{\mathrm{x}} \mathrm{Ga}_{1-\mathrm{x}}$ As hillocks.

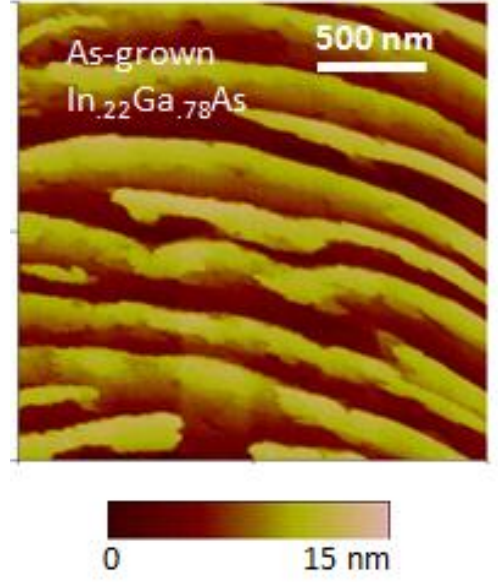

(a)

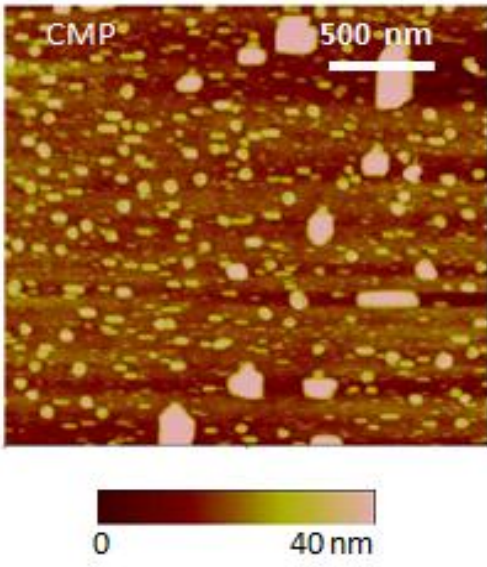

(b)

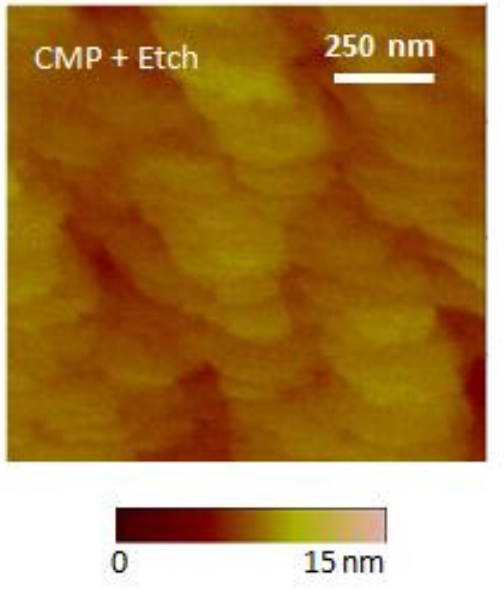

(c)

Fig 1. AFM measurements showing surface morphology of MBLs post annealing treatment at $630^{\circ} \mathrm{C}$ (a) As-grown, (b) CMP treatment applied, (c) CMP and Wet-etching treatment applied

The In enrichment changes the local lattice parameter and subsequent growth leads to lattice mismatch-based defects propagating into the subsequently grown layer. This is evident from the bright field diffraction contrast TEM image of an MOVPE-grown strained-layer superlattice with $\mathrm{In}_{\mathrm{x}} \mathrm{Ga}_{1-\mathrm{x}} \mathrm{As}$ wells/ $\mathrm{Al}_{\mathrm{y}} \mathrm{In}_{1-\mathrm{y}} \mathrm{As}$ barriers on top of the MBL [5]. An increase in the full width half maximum (FWHM) of the SL XRD fringes when compared to the regrowth on an as-grown MBL was also observed $[5,6]$.

The In segregation found after annealing was mitigated by employing a post CMP wet-chemical etch. Prior to growth on the MBL, the MBL was wet-etched in a $\mathrm{H}_{2} \mathrm{O}: \mathrm{H}_{3} \mathrm{PO}_{4}: \mathrm{H}_{2} \mathrm{O}_{2}(38: 1: 1)$ solution for one minute which removes residual damage from the surface that had been introduced by the CMP [5]. As can be seen in Fig. 1(c), the CMP/wet-etched MBL has a 
different morphology but no strong indication of the In-enriched surface features after annealing at a high temperature of $630^{\circ} \mathrm{C}$.

\subsection{QCL active region design and regrowth}

The QCL was designed to emit at $3.39 \mu \mathrm{m}$ employing a single phonon resonance depopulation scheme [6]. This design requires a substrate with a lattice constant of $0.574 \mathrm{~nm}$, which corresponds to relaxed $\operatorname{In}_{0.22} \mathrm{Ga}_{0.78} \mathrm{As}$. The $\mathrm{MBL}$ cap layer was designed to have this composition. The active region consists of a 10-period SL consisting of constant composition but varying thicknesses of tensile-strained AlAs barriers $(\Delta \mathrm{a} / \mathrm{a} \sim 1.42 \%$ each) and compressivelystrained $\operatorname{In}_{0.45} \mathrm{Ga}_{0.55} \mathrm{As}$ wells $(\Delta \mathrm{a} / \mathrm{a} \sim 1.61 \%$ each). The important design characteristics of this QCL are a high $\mathrm{E}_{43}$ value $(84.4 \mathrm{meV})$ which minimizes carrier leakage from upper laser level [7] and a large $\mathrm{E}_{2}-\mathrm{E}_{\mathrm{gn}}$ value $(226.4 \mathrm{meV})$ which minimizes the backfilling current density in the lower laser levels of a period resulting from thermal excitation from the ground state of the injector for the next period.

As a measure of the suitability of these MBL substrates for QCL use, growth of ten stages of this QCL design was carried out on the CMP and wet-etched MBL surface. Optical Images obtained using the Nomarski microscope demonstrate that the surface morphology is significantly smoother than the as-grown MBL for the test structure regrowth (Fig. 2). It is observed that the applied polishing procedure is effective in preparing a virtual substrate appropriate for regrowth of structures with strained layers, as is also evident from the TEM and XRD data. 


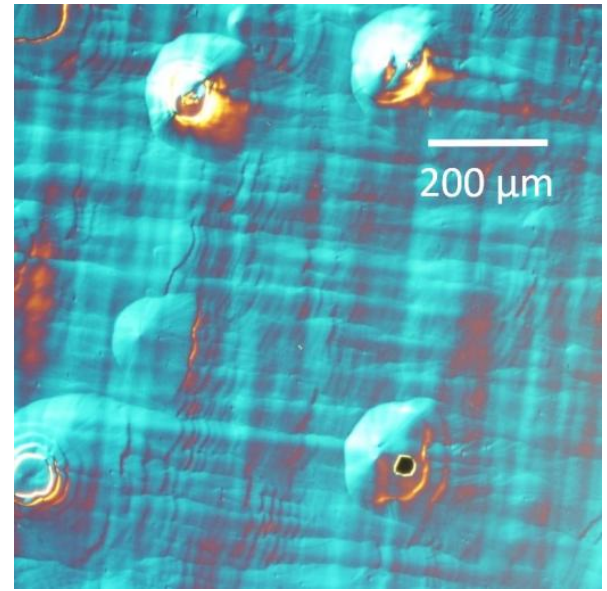

(a)

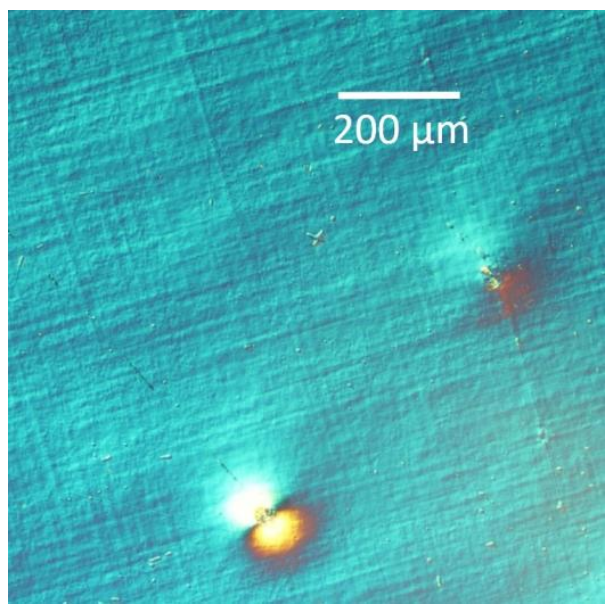

(b)

Fig 2. Optical Images, using Nomarski interference contrast, depicting surface morphology of (a) As-grown MBL, (b) 10-stage QCL active region regrowth on a surface-optimized MBL

To assess the structural characteristics of the superlattice region grown on the MBL, grown layers were imaged using diffraction contrast TEM of $\langle 110\rangle$ cross-sectional specimens. The image shown in Fig. 3(a) portrays the ten stage stack of the QCL active region grown on the MBL. Dislocations originating in the MBL cap layer are seen propagating through the regrown layers. The dislocation density for this sample is estimated to be $1 \times 10^{5} \mathrm{~cm}^{-2}$ from the etch pits observed in dark field images obtained with an optical microscope. Fig. 3(b) is a higher magnification image of a few stages showing distinct contrast to the well and barrier layers suggesting sharp and uniform interfaces. Fig. 3(c) is a phase contrast TEM image providing atomic scale detail. This image was used to calculate the individual layer thicknesses which are subsequently used to fit the XRD experimental data (Table I). 


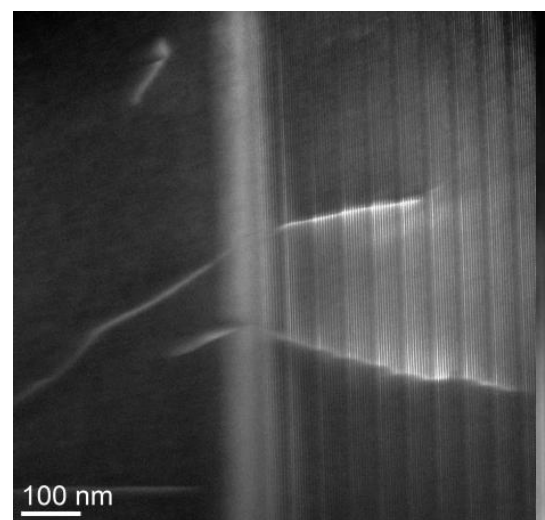

(a)

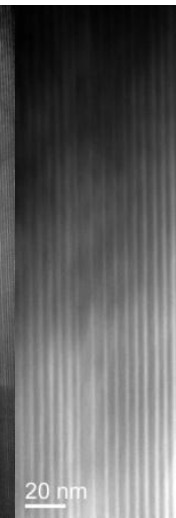

(b)

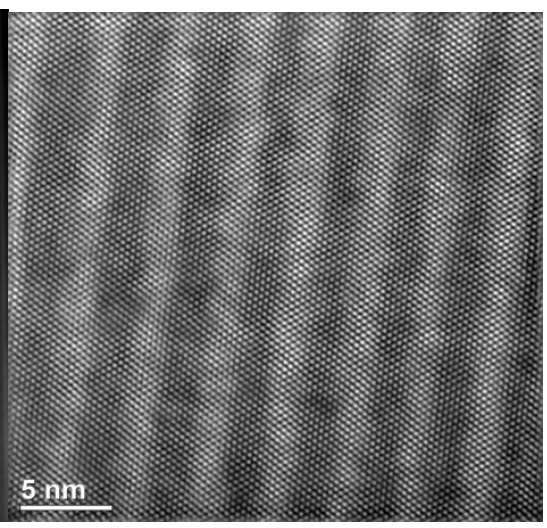

(c)

Fig 3. TEM images: (a) $\{220\}$ dark- field image of ten stages of QCL active region regrowth on an MBL, showing dislocations propagating from the cap layer through the regrown SL layers, (b) Higher magnification image of (a), (c) Phase contrast high resolution image providing atomic detail within a stage

\begin{tabular}{|l|l|l|l|l|l|l|l|l|l|l|l|l|l|l|l|l|l|l|l|l|}
\hline Target thickness & $\mathbf{2 5}$ & 16 & $\mathbf{2 4}$ & 17 & $\mathbf{2 1}$ & 18 & $\mathbf{2 0}$ & $\mathbf{2 1}$ & $\mathbf{1 9}$ & 21 & $\mathbf{1 8}$ & 18 & $\mathbf{1 7}$ & 28 & $\mathbf{1 2}$ & 13 & $\mathbf{3 9}$ & 10 & $\mathbf{3 3}$ & 16 \\
\hline Actual thickness & $\mathbf{2 6}$ & 18 & $\mathbf{2 3}$ & 20 & $\mathbf{2 0}$ & 18 & $\mathbf{2 0}$ & 20 & $\mathbf{1 8}$ & 18 & $\mathbf{1 8}$ & 18 & $\mathbf{1 8}$ & 23 & $\mathbf{1 2}$ & 15 & $\mathbf{4 1}$ & 18 & $\mathbf{3 5}$ & 18 \\
\hline
\end{tabular}

Table I. Comparison of the target thicknesses for one stage of the active region design with the actual thickness values calculated from Fig. 3(c). All thickness values are in Angstroms. Bold numbers indicate wells and others indicate barriers in the QCL test structure.

The high-resolution X-ray diffraction pattern consisting of a reciprocal space map (RSM) of the (004) reflection is shown in Fig. 4(a). The well-defined superlattice fringes possess a narrow FWHM within the RSM indicating that the layers are uniform with relatively sharp intefaces. Fig. 4(b) shows the integrated mosaic intensity along the $\omega$-axis at each point on the $2 \theta$ axis. This simulates a conventional rocking curve taken in a $\omega-2 \theta$ two geometry. The integrated line is shown as the upper solid line while the lower dashed line is a simulation portraying the best 
fit. The best fit simulation is obtained by using $1.15 \times$ TEM thickness for the wells and 0.8xTEM thickness for the barriers. Upto a $20 \%$ error in the calculated thicknesses is well within the acceptable range of uncertainty for deriving such layers from the TEM micrograph. This uncertainty amounts to +/- one monolayer which is easily accounted for in the abruptness of the interfaces.

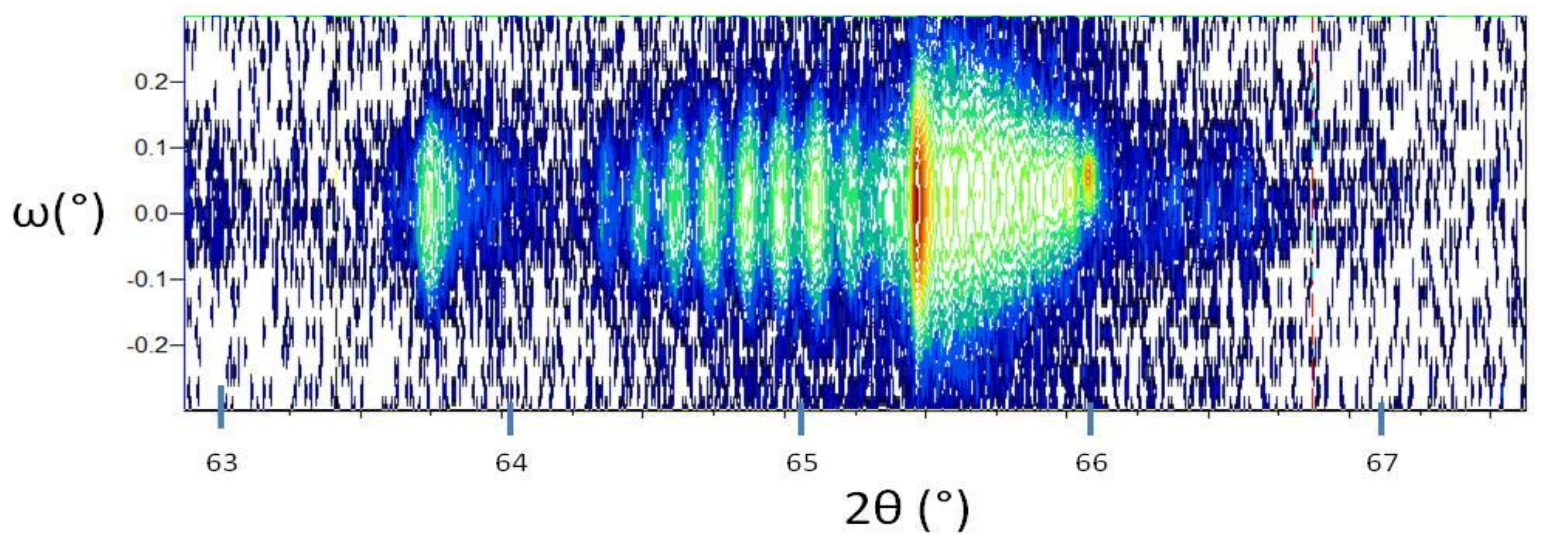

(a)

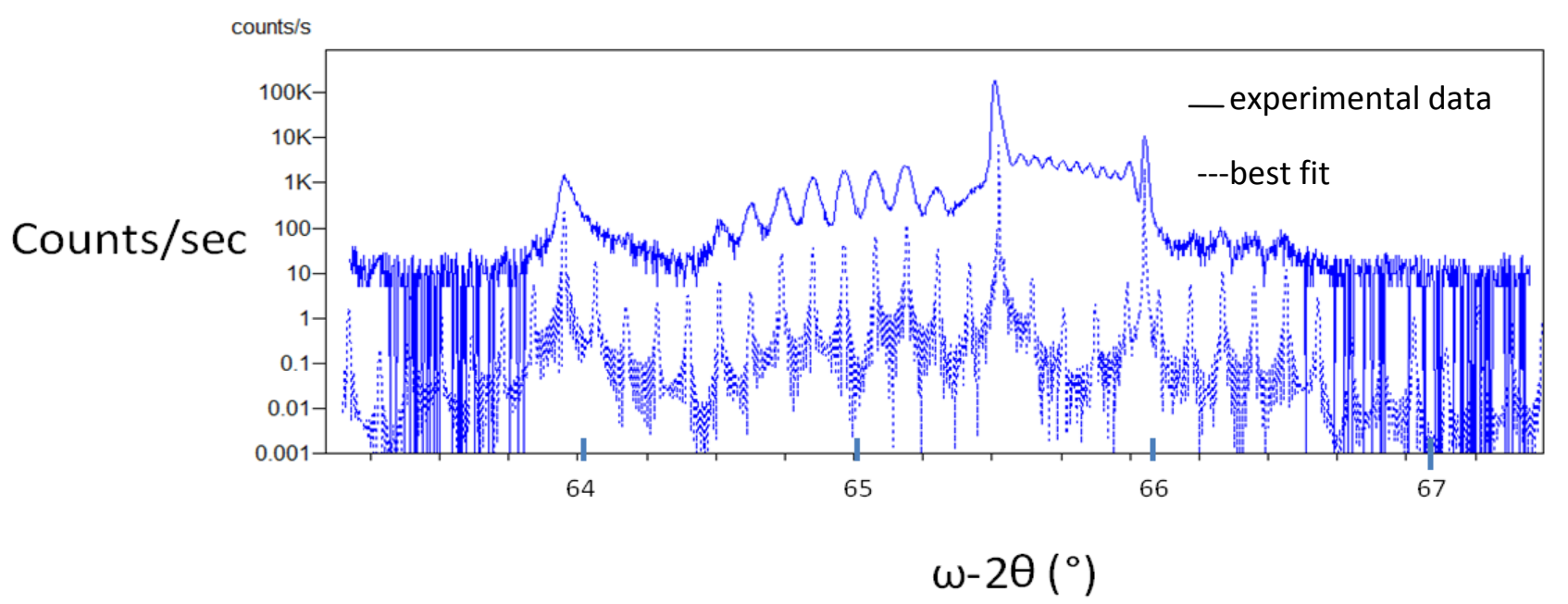

(b)

Fig 4. HR-XRD scans (a) (004) $\omega-2 \theta$ RSM , (b) (004) $\omega$-2 $2 \theta$ line scan (solid) compared with best fit simulation (dashed) 


\section{Conclusions}

An HVPE-grown MBL was used to access a substrate lattice parameter which could not be obtained by the use of a conventional binary semiconductor substrate. This change in lattice parameter allows for the use of barrier and well compositions which would be otherwise prohibited due to excessive strain if grown on a conventional substrate, thus enables the development of a short-emission-wavelength QCL of potentially significantly improved performance $[2,6]$. CMP and wet-chemical etching is found to be effective in preparing the MBL surface for growth of the QCL structure. Successful MOVPE growth of ten stages of a QCL active region on such a surface has been achieved with high structural fidelity.

\section{Acknowledgements}

This work is supported by The National Science Foundation, Materials Research and Engineering Center (DMR-1121288) and Partners for Innovation (PFI) 1317292, and Navy STTR N68335-11-C-0432.

\section{References}

[1] M. Arai, K. Nakashima, T. Fujisawa, T. Tadokoro, W. Kobayashi, M. Yuda, Y. Kondo, IEEE Journal of Selected Topics in Quantum Electronics., 15(3) (2009), pp.724-730

[2] L. J. Mawst, J. D. Kirch, T.W. Kim, T. Garrod, C. Boyle, D. Botez, B. Zutter, K. Schulte, T. F. Kuech, P. M. Bouzi, C. F. Gmachl, T. Earles, IET Optoelectronics, 8 (2014), pp. 25-32.

[3] R. Beanland, D.J. Dunstan, and P.J. Goodhew, Adv. Phys. 45 (1996), p.87

[4] K. Schulte, T. Garrod, T.W. Kim, J.D. Kirch, S. Ruder, L.J. Mawst and T.F. Kuech, Journal of Crystal Growth., 370 (2013), pp. 293-298

[5] B Zutter, K Schulte, E Lewis, T Kim, A. Rajeev, T Earles, S Babcock, L Mawst, T Kuech, Surface Crystalline Defects in $\mathrm{In}_{\mathrm{x}} \mathrm{Ga}_{1-\mathrm{x}} \mathrm{As}$ Introduced by Polishing Methods, TMS Electronics Materials Conference, Santa Barbara, CA, June 25-27 ${ }^{\text {th }}, 2014$ 
[6] L.J. Mawst, A. Rajeev, J.D. Kirch, T.W. Kim, D. Botez, B. Zutter, P. Buelow., K. Schulte, T.F. Kuech, A. Wood, S.E. Babcock, T. Earles, Proceedings of SPIE--the International Society for Optical Engineering, 9370 (2015), 93700E.

[7] D. Botez, S. Kumar, J. C. Shin, L. J. Mawst, I. Vurgaftman and J. R. Meyer, Appl. Phys. Lett., 97 (2010), p. 071101; Erratum: Appl. Phys. Lett., 97 (2010), p. 199901. 